Tropical Journal of Pharmaceutical Research January 2020; 19 (1): 89-94

ISSN: $1596-5996$ (print); 1596-9827 (electronic)

(C) Pharmacotherapy Group, Faculty of Pharmacy, University of Benin, Benin City, 300001 Nigeria.

\title{
Mangiferin ameliorates insulin resistance in a rat model of polycystic ovary syndrome via inhibition of inflammation
}

\author{
Qiaohong Qian, Minjie Tang, Xinrong Li, Qi Cao*, Zhiling Zhu \\ Department of Integrative Medicine, Obstetrics and Gynecology Hospital, Fudan University, Shanghai 200011, China \\ ${ }^{*}$ For correspondence: Email: qicao@fudan.edu.cn; Tel: +86-021-33189900
}

Sent for review: 11 October 2019

Revised accepted: 27 December 2019

\begin{abstract}
Purpose: To examine the effect of mangiferin on insulin resistance (IR) in a rat polycystic ovary syndrome (PCOS) model.

Methods: The rat PCOS model was established via subcutaneous injection of $6 \mathrm{mg} / \mathrm{kg}$ of dehydroepiandrosterone (DHEA), and mangiferin was orally administered. Body and ovarian weights were recorded. Serum levels of glucose, insulin, and related inflammatory cytokines were evaluated by quantitative real-time polymerase chain reaction (qRT-PCR) and enzyme-linked immunosorbent assay, while the expression levels of key proteins were analyzed by western blotting.

Results: DHEA significantly increased ovarian weight and the ratio of ovarian weight/body weight $(p<$ $0.001)$, while mangiferin treatment decreased them $(p<0.001)$. Mangiferin also lowered DHEA-induced enhancements in serum glucose and insulin levels $(p<0.001)$. The mRNA and, expression and concentrations of inflammatory cytokines (interleukin-6(IL-6), interleukin-1 $\beta$ (IL-1 $\beta)$ and tumor necrosis factor- $\alpha(T N F-\alpha))$ were also significantly reduced by mangiferin treatment $(p<0.001)$. Furthermore, mangiferin suppressed phosphorylation of nuclear factor-kappa $B$ (NF-KB) but increased the phosphorylation of protein kinase $B(A K T, p<0.001)$.

Conclusion: These results reveal that mangiferin not only decreases inflammatory cytokine levels by regulating NF-KB signaling pathway but also ameliorates IR in a rat PCOS model via regulating AKT signaling pathway. Thus, mangiferin is a potential therapeutic strategy for the management of PCOS.
\end{abstract}

Keywords: Polycystic ovary syndrome, Mangiferin, Inflammation, Insulin resistance, NF-KB, AKT

\begin{abstract}
This is an Open Access article that uses a fund-ing model which does not charge readers or their institutions for access and distributed under the terms of the Creative Commons Attribution License (http://creativecommons.org/licenses/by/4.0) and the Budapest Open Access Initiative (http://www.budapestopenaccessinitiative.org/read), which permit unrestricted use, distribution, and reproduction in any medium, provided the original work is properly credited.
\end{abstract}

Tropical Journal of Pharmaceutical Research is indexed by Science Citation Index (SciSearch), Scopus, International Pharmaceutical Abstract, Chemical Abstracts, Embase, Index Copernicus, EBSCO, African Index Medicus, JournalSeek, Journal Citation Reports/Science Edition, Directory of Open Access Journals (DOAJ), African Journal Online, Bioline International, Open-J-Gate and Pharmacy Abstracts

\section{INTRODUCTION}

Polycystic ovary syndrome (PCOS), a gynecological endocrine disorder, is characterized by insulin resistance (IR) [1]. Although lifestyle changes help alleviate PCOS, there is still devoid of cure [2]. Therefore, it is necessary to develop more effective therapeutic drugs and strategies for the treatment of PCOS.
Mangiferin was firstly isolated from the leaves and bark of the mango tree (Mangifera indica), and it can also be found in mango peels and kernels. Mangiferin has also been found in Iris unguicularis, the rhizomes of Anemarrhena asphodeloides, leaves of Bombax ceiba, and in the genera Salacia and Cyclopia [3]. One study showed that mangiferin can function as a 
bioactive compound to regulate blood glucose levels and can help treat lifestyle-related disorders, such as IR [3]. Mangiferin can effectively increase the uptake of glucose by 3T3-L1 adipocytes [4]. Moreover, mangiferin can ameliorate metabolic disorders caused by high lipid levels, increase the utilization rate of glucose, and maintain the physiological homeostasis of insulin, thus protecting against IR [5]. In addition, the anti-inflammatory effects of mangiferin have been widely reported.

Mangiferin has been shown to reduce the inflammatory response of mouse macrophages stimulated by lipopolysaccharide by inactivation of nucleotide-binding oligomerization domain-like receptor pyrin domain-containing protein 3 inflammasome [6]. Mangiferin also reduces the level of inflammation in rats after spinal cord injury [7]. However, the effects of mangiferin on PCOS-induced IR and inflammation have not been reported previously, and the mechanism is still unclear.

This study aimed to investigate the effects of mangiferin on IR and inflammation in a rat PCOS model, and reveal the underlying mechanism. The present study may contribute to the development of novel therapeutics for the treatment of human PCOS.

\section{EXPERIMENTAL}

\section{Animal experimental model}

Three-week-old SPF-grade female Wistar rats ( $=60,35-40 \mathrm{~g}$ ), were used. Each rat was housed separately and provided a standard pellet diet and water based on the "Guide for the Care and Use of Laboratory Animals" [8] and approved by the Ethics Committee of Obstetrics and Gynecology Hospital, Fudan University (approval no. 2017FD046). The rats were assigned to two groups: 48 rats were subcutaneously injected with DHEA (Sigma, St. Louis, Missouri, USA) (6 $\mathrm{mg} / \mathrm{kg}$ body weight/day, dissolved in $0.2 \mathrm{~mL}$ sesame oil) for 28 days and 12 control rats were subcutaneously injected with $0.2 \mathrm{~mL}$ sesame oil per day for 28 days.

Twenty days later, rats in the DHEA treatment group were subjected to exfoliative vaginal cytology to identify rats with PCOS, as described previously [9]. Forty rats with PCOS were then assigned to four groups (10 rats per group): the DHEA group, the $10 \mathrm{mg} / \mathrm{kg}$ mangiferin group, 20 $\mathrm{mg} / \mathrm{kg}$ mangiferin group, and $30 \mathrm{mg} / \mathrm{kg}$ mangiferin group. The DHEA and control groups were orally fed $0.01 \mathrm{~mL} / \mathrm{g}$ saline per day in place of mangiferin. After treatment for 4 weeks, the body weights of rats were recorded. The rats were then anesthetized with $1.5 \%$ pentobarbital sodium (40 mg/kg body weight). Blood was collected from the orbital vein, and ovarian weights were recorded.

\section{Determination of glucose, insulin and inflammatory cytokine levels}

For the detection of glucose and insulin levels, the rats were fed a $3-\mathrm{g} / \mathrm{kg}$ glucose solution (Sigma) by oral gavage prior to anesthesia. Blood was collected 30, 60, 90, and $120 \mathrm{~min}$ after glucose administration using glucometry (Cygnus, Redwood City, CA, USA). Blood collected $120 \mathrm{~min}$ after glucose administration was used for the detection of insulin and inflammatory cytokine (interleukin-6(IL-6), interleukin-1 $\beta$ (IL-1 $\beta$ ) and tumor necrosis factor- $\alpha$ (TNF- $\alpha)$ ) levels using an enzyme-linked immunosorbent assay kit (Sigma).

\section{Quantitative real-time polymerase chain reaction}

Total RNA was extracted from rat ovaries and reverse-transcribed into cDNA using the miScript Reverse Transcription kit (Qiagen, Hilden, Germany). cDNA was then amplified using SYBR1 Premix Ex Taq $^{\text {TM }}$ II (Takara, Shiga, Japan). GAPDH was used as the endogenous control. The primer sequences are shown in Table 1.

Table 1: Primer sequence for PCR

\begin{tabular}{ll}
\hline ID & Sequence (5'-3') \\
\hline GAPDH F & TGTTCGTCATGGGTGTGAAC \\
GAPDH R & ATGGCATGGACTGTGGTCAT \\
IL-6 F & ATGAACTCCTTCTCCACAAGC \\
IL-6 R & GTTTTCTGCCAGTGCCTCTTTG \\
IL-1 $\beta \mathrm{F}$ & CACCTTCTTTTCCTTCATCTTTG \\
IL-1 $\mathrm{R}$ & GTCGTTGCTTGTCTCTCCTTGTA \\
TNF- $\alpha \mathrm{F}$ & ACTGAACTTCGGGGTGATTG \\
TNF- $\alpha \mathrm{R}$ & GCTTGGTGGTTTGCTACGAC \\
\hline
\end{tabular}

\section{Western blot}

Proteins were extracted from rat ovaries via RIPA lysis buffer (Beyotime, Beijing, China), subjected to $10 \%$ SDS-PAGE, and transferred to a PVDF membrane. The membrane was blocked in PBST containing 5\% BSA for $2 \mathrm{~h}$ and then probed with rabbit antibodies to p65, p-p65 (1:1,500, Abcam, Cambridge, MA, USA), AKT, pAKT (1:2,000, Abcam), and GAPDH (1:3,000, Abcam) overnight at $4{ }^{\circ} \mathrm{C}$. The membrane was then washed with TBST and incubated with horseradish peroxidase-conjugated secondary antibody (1:5,000; Abcam) for $1 \mathrm{~h}$. 
Immunoreactivities were detected by enhanced chemiluminescence (KeyGen, Nanjing, China).

\section{Statistical analysis}

The results were expressed as mean \pm SEM of at least three independent experiments. Statistical analyses were performed using GraphPad Prism software (GraphPad Prism Software Inc., San Diego, USA) and one-way analysis of variance. $p<0.05,0.01$, or 0.001 were considered statistically significant.

\section{RESULTS}

\section{Mangiferin improves ovarian function in rats with PCOS}

In the DHEA induced PCOS rat model, the body weight (Figure 1A) and the ratio of ovarian weight/body weight were significantly increased (Figure 1B) $(p<0.001)$. However, these levels were significantly decreased by the administration of mangiferin compared with the DHEA group $(p<0.001)$. Interestingly, the effects of mangiferin were dose-dependent, showing that $30 \mathrm{mg} / \mathrm{kg}$ mangiferin treatment group had the lowest body weight and the ratio of ovarian weight/body weight. Therefore, mangiferin might improve the ovarian function of DHEA induced PCOS rats.
A

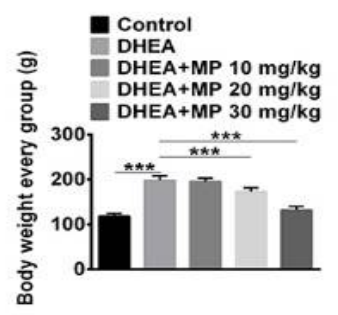

B

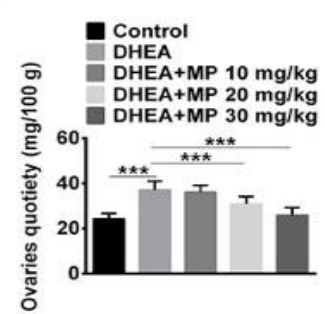

Figure 1: Mangiferin decreased body weight and the ratio of ovarian weight/body weight in DHEA induced PCOS rats. (A) Mangiferin decreased body weight of rats with PCOS induced by DHEA. (B) Mangiferin decreased the ratio of ovarian weight/body weight in rats with PCOS induced by DHEA. ${ }^{* * *} p<0.001$, DHEA vs. Control or $10,20,30 \mathrm{mg} / \mathrm{kg}$ mangiferin vs. DHEA

\section{Mangiferin decreases the levels of glucose and insulin in rats with PCOS}

To further investigate the effect of mangiferin on PCOS, serum glucose and insulin levels were measured. First, a rat blood glucose release curve at different time points was generated and found that blood glucose release was time dependent: the glucose level at 30 min after the oral administration of glucose was the highest, and the glucose level at $120 \mathrm{~min}$ after the oral administration of glucose was the lowest (Figure 2 A). DHEA (dehydroepiandrosterone) treatment significantly increased glucose levels as compared to the control (Figure $2 \mathrm{~A})(p<0.001)$. And the administration of mangiferin decreased blood glucose levels (Figure $2 \mathrm{~A}$ ) as compared with the DHEA group. Similarly, DHEA treatment significantly increased insulin levels compared to the control (Figure 2 B) $(p<0.001)$, and the administration of mangiferin decreased insulin levels (Figure $2 \mathrm{~A}$ ) as compared with the DHEA group. These results revealed that mangiferin could decrease the levels of glucose and insulin (two characteristic of IR) in rats with PCOS.
A

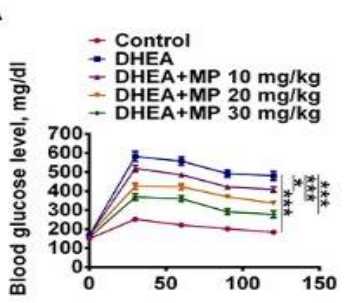

A

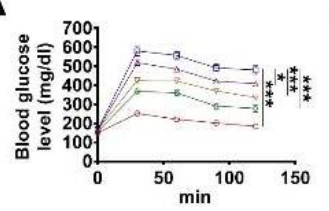

B

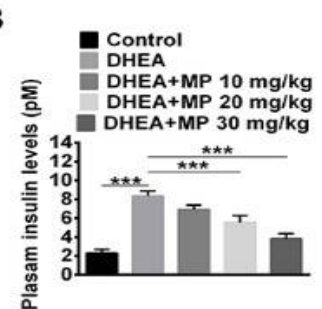

B

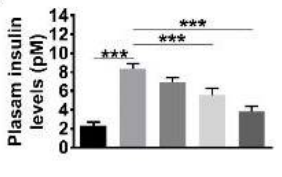

Figure 2: Mangiferin decreases the levels of glucose and insulin in rats with PCOS. (A) Mangiferin decreased blood glucose level of rats with PCOS induced by DHEA. ${ }^{-}$: Control, $\square$ : DHEA, ${ }^{\triangle}$ : DHEA+MF $10 \mathrm{mg} / \mathrm{kg},{ }^{-}$: DHEA+MF $20 \mathrm{mg} / \mathrm{kg},{ }^{\diamond}$ : DHEA+MF $30 \mathrm{mg} / \mathrm{kg}$. (B) Mangiferin decreased insulin levels of rats with PCOS induced by DHEA. $\mathbf{a}$ : Control, ㅁ: DHEA, ㅁ: DHEA+MF $10 \mathrm{mg} / \mathrm{kg}, \square:$ DHEA+MF $20 \mathrm{mg} / \mathrm{kg}$, : : DHEA+MF $30 \mathrm{mg} / \mathrm{kg} .{ }^{*} p<$ $0.05,{ }^{* * *} p<0.001$ DHEA vs. Control or $10,20,30$ $\mathrm{mg} / \mathrm{kg}$ mangiferin vs. DHEA.

\section{Mangiferin ameliorates inflammation in rats with PCOS}

The effects of mangiferin on the expression of inflammatory cytokines were then determined. Results showed that DHEA treatment significantly increased the mRNA expression (Figure $3 \mathrm{~A}$ ) and concentrations (Figure $3 \mathrm{~B}$ ) of inflammatory cytokines, such as IL-6/1 $\beta$ and TNF- $\alpha$, compared to control $(p<0.001)$. Moreover, the administration of mangiferin decreased the mRNA expression (Figure $3 \mathrm{~A}$ ) and concentrations (Figure $3 \mathrm{~B}$ ) of $\mathrm{IL}-6 / 1 \beta$ and TNF- $\alpha$ as compared with the DHEA group. Therefore, mangiferin could ameliorate inflammation in rats with PCOS. 
A
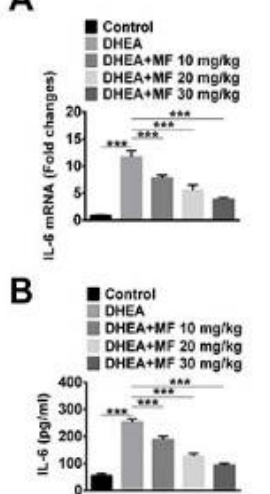
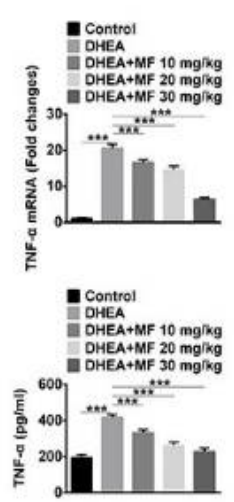
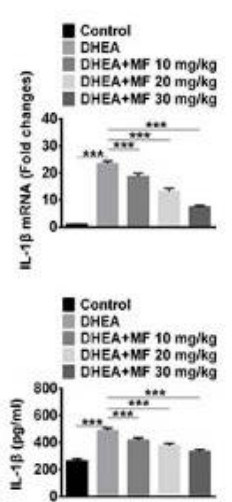

Figure 3: Mangiferin decreases the secrection of inflammatory cytokines in rats with PCOS. (A) Mangiferin decreased the mRNA expression of IL-6/1 $\beta$ and TNF- $\alpha$ in rats with PCOS induced by DHEA. (B) Mangiferin decreased the concentrations of IL-6/1 $\beta$ and TNF- $\alpha$ in rats with PCOS induced by DHEA. ${ }^{* * *} p$ $<0.001$, DHEA vs. Control or $10,20,30 \mathrm{mg} / \mathrm{kg}$ mangiferin vs. DHEA

\section{Mangiferin regulates the NF-KB and AKT pathways in rats with PCOS}

Western blot analysis revealed that DHEA treatment had no significant effects on the protein expression of NF-KB (p65), but the phosphorylation of $\mathrm{p} 65$ (p-p65) and the $\mathrm{p}$ p65/p65 ratio were increased by DHEA (Figure 4). Moreover, the administration of mangiferin decreased the protein expression of p-p65 and the $p-p 65 / p 65$ ratio (Figure 4). Furthermore, DHEA and mangiferin had no significant effects on the expression of AKT, but the phosphorylation of AKT ( $p-A K T)$ and the $p$ AKT/AKT ratio were decreased by DHEA (Figure 4). In contrast, the administration of mangiferin increased the levels of $\mathrm{p}$-AKT and the $\mathrm{p}$ AKT/AKT ratio (Figure 4). Thus, mangiferin inactivated the NF-KB signaling pathway and activated the AKT pathway in rats with PCOS.

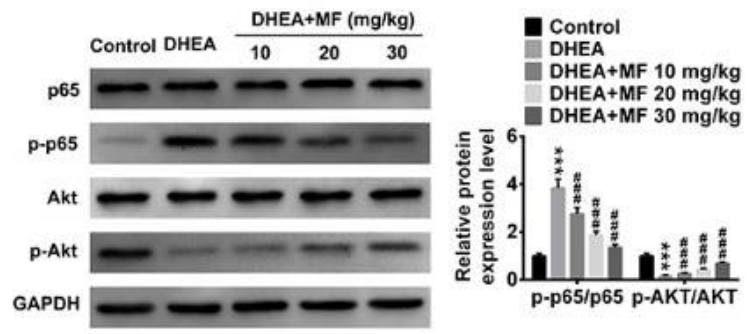

Figure 4: Mangiferin decreased $p-65$ and increased $p$ Akt in rats with PCOS. Mangiferin decreased the levels of $p-p 65$ and $p-A K T$ in rats with PCOS induced by DHEA, but had no significant effects on protein expression of p65 and AKT. ${ }^{* *} p<0.001$, DHEA vs. Control, ${ }^{\# \#} p<0.001,10,20,30 \mathrm{mg} / \mathrm{kg}$ mangiferin vs. DHEA

\section{DISCUSSION}

Bioactive compound quercetin has been shown to restrain inflammatory responses to prevent PCOS with fewer side effects than metformin (a conventional treatment for PCOS) [10]. Recently, mangiferin, also known as 1, 3, 6, 7tetrahydroxyxanthone, (chemical name: $2-\beta-D-$ glucopyranosyl-1, $3, \quad 6, \quad$ 7-tetrahydroxy-9Hxanthen-9-one; chemical formula: $\mathrm{C}_{19} \mathrm{H}_{18} \mathrm{O}_{11}$ ), is a xanthone found in higher plants, including mango fruit [11]. Recent pharmacological studies have indicated that mangiferin elicits antioxidant, antidiabetic, and immunomodulatory effects $[12,13]$. Therefore, the protective role of mangiferin against PCOS and the underlying mechanism were investigated in this study using a rat $\mathrm{PCOS}$ model.

DHEA, a steroid hormone, is widely used to induce PCOS in rats for research [14]. In the present study, a rat PCOS model were successfully established, as shown by increases in body and ovarian weights, and the ratio of ovarian weight/body weight. Moreover, IR, as a leading cause of PCOS [15], was also induced by DHEA treatment, as shown by increases in serum glucose and insulin levels. In line with previous research showing that mangiferin can ameliorate PCOS by decreasing IR [16], the present study demonstrated that mangiferin not only decreased the ratio of ovarian weight/body weight to improve ovarian function, but also decreased serum glucose and insulin levels to ameliorate IR. The anti-IR effect of mangiferin in 3T3-L1 adipocytes was revealed by the inhibition of both glucose uptake and insulin signaling [17]. During insulin signaling, insulin binds to its receptor, phosphorylates the insulin receptor substrate-1 (IRS-1), and recruits the phosphatidylinositol 3-kinase to activate AKT and other downstream targets [18]. The activation of AKT facilitates the translocation of glucose transporter type 4 and stimulates glucose uptake [19]. Therefore, the AKT signaling pathway exerts a vital role in the regulation of IR. In the present study, mangiferin increased levels of $p$ AKT and the $p$-AKT/AKT ratio, thus activating AKT signaling to ameliorate IR in rats with PCOS; this same mechanism is utilized by quercetin [20]. However, due to the regulation of other complicated cellular functions, AKT signaling may affect PCOS by a mechanism other than its effects on IR, which requires further study.

Inflammation is considered a key contributor to the pathogenesis of PCOS [21]. In line with a previous study in which mangiferin decreased inflammation [22], this study revealed that 
mangiferin decreased the expression of inflammatory cytokines IL-6/1 $\beta$ and TNF- $\alpha$ induced by DHEA, thus ameliorating inflammatory responses in rats with PCOS. Mangiferin is known to activate adenosine 5monophosphate-activated protein kinase [17], which blocks NF-KB signaling by the inhibition of IL-6/1 $\beta$ and TNF- $\alpha$, thus reducing inflammation [23]. Therefore, the involvement of NF-KB signaling pathway in the effects of mangiferin on inflammatory responses in PCOS is verified. Here, results revealed that DHEA induced chronic inflammation by increasing the p-p65/p65 ratio, thus activating the NF-kB signaling pathway. However, mangiferin decreased the $p$ p65/p65 ratio, thus blocking NF-KB signaling and the expressions of inflammatory cytokines. In PCOS patients, glucose may induce oxidative stress which stimulates an inflammatory response, and the inflammatory cytokine TNF- $\alpha$ has been shown to increase the serine phosphorylation of IRS-1 as a mediator of IR [24]. Therefore, inflammation may underpin IR in PCOS [25]. Quercetin was found to ameliorate IR in PCOS by improving the inflammatory microenvironment [10] and mangiferin was also found to ameliorate IR by inhibiting inflammation [17]. The present study showed that mangiferin ameliorated IR in a rat PCOS model by inhibiting inflammation.

\section{CONCLUSION}

The present study demonstrates that mangiferin improves ovarian function, ameliorates IR, and inhibits inflammatory responses in rats with PCOS. Moreover, the protective effects of mangiferin are closely associated with the inhibition of NF-KB signaling and activation of AKT signaling. Thus, the findings provide a new insight into the relationship between the components of mangiferin/NF-KB/AKT regulatory axis and PCOS, suggesting the potential application of mangiferin as a new therapeutic strategy for PCOS.

\section{DECLARATIONS}

\section{Acknowledgement}

This work was supported by Special Project of Traditional Chinese Medicine Scientific Research of Shanghai Municipal Health and Family Planning Commission (grant no. 2016LP018).

\section{Competing interests}

The authors state that there is no conflict of interest to disclose with regard to this work.

\section{Contribution of authors}

We declare that this work was done by the researchers listed in this article. All liabilities related to the content of this article will be borne by the authors. Qi Cao and Zhiling Zhu designed all the experiments and revised the paper. Qiaohong Qian and Minjie Tang performed the experiments, Xinrong Li wrote the manuscript.

\section{Open Access}

This is an Open Access article that uses a funding model which does not charge readers or their institutions for access and distributed under the terms of the Creative Commons Attribution License (http://creativecommons.org/licenses/by/ 4.0) and the Budapest Open Access Initiative (http://www.budapestopenaccessinitiative.org/rea d), which permit unrestricted use, distribution, and reproduction in any medium, provided the original work is properly credited.

\section{REFERENCES}

1. Hong $Y$, Yin $Y$, Tan $Y$, Hong $K$, Jiang $F$, Wang YJTJoPR. Effect of quercetin on biochemical parameters in letrozole-induced polycystic ovary syndrome in rats. 2018; 17(9): 1783-1788.

2. Patel S. Polycystic ovary syndrome (PCOS), an inflammatory, systemic, lifestyle endocrinopathy. J Steroid Biochem Mol Biol 2018; 182: 27-36.

3. Imran M, Arshad MS, Butt MS, Kwon JH, Arshad MU, Sultan MT. Mangiferin: a natural miracle bioactive compound against lifestyle related disorders. Lipids Health Dis 2017; 16(1): 84.

4. Kumar BD, Krishnakumar K, Jaganathan SK, Mandal M. Effect of Mangiferin and Mahanimbine on Glucose Utilization in 3T3-L1 cells. Pharmacogn Mag 2013; 9(33): $72-75$.

5. Apontes $P$, Liu Z, Su K, Benard O, Youn DY, Li X, Li W, Mirza RH, Bastie CC, Jelicks $L A$ et al. Mangiferin stimulates carbohydrate oxidation and protects against metabolic disorders induced by high-fat diets. Diabetes 2014; 63(11): 3626-3636.

6. Bulugonda RK, Kumar KA, Gangappa D, Beeda H, Philip $G H$, Muralidhara Rao D, Faisal SM. Mangiferin from Pueraria tuberosa reduces inflammation via inactivation of NLRP3 inflammasome. Sci Rep 2017; 7: 42683.

7. Luo Y, Fu C, Wang Z, Zhang Z, Wang H, Liu Y. Mangiferin attenuates contusive spinal cord injury in rats through the regulation of oxidative stress, inflammation and the Bcl2 and Bax pathway. Mol Med Rep 2015; 12(5): 7132-7138.

8. Hajiaghaalipour F, Kanthimathi MS, Abdulla MA, Sanusi $J$. The Effect of Camellia sinensis on Wound Healing Potential in an Animal Model. Evid Based Complement Alternat Med 2013; 2013: 386734.

Trop J Pharm Res, January 2020; 19(1): 93 
9. Yu J, Zhai D, Hao L, Zhang D, Bai L, Cai Z, Yu C. Cryptotanshinone Reverses Reproductive and Metabolic Disturbances in PCOS Model Rats via Regulating the Expression of CYP17 and AR. Evid Based Complement Alternat Med 2014; 2014: 670743.

10. Wang Z, Zhai D, Zhang D, Bai L, Yao R, Yu J, Cheng $W$, Yu C. Quercetin Decreases Insulin Resistance in a Polycystic Ovary Syndrome Rat Model by Improving Inflammatory Microenvironment. Reprod Sci 2017; 24(5): 682-690.

11. Dar A, Faizi S, Naqvi S, Roome T, Zikr-ur-Rehman S, Ali $M$, Firdous S, Moin ST. Analgesic and antioxidant activity of mangiferin and its derivatives: the structure activity relationship. Biol Pharm Bull 2005; 28(4): 596 600.

12. Na L, Zhang Q, Jiang S, Du S, Zhang W, Li Y, Sun C, Niu $Y$. Mangiferin supplementation improves serum lipid profiles in overweight patients with hyperlipidemia: a double-blind randomized controlled trial. Sci Rep 2015; 5: 10344.

13. Niu Y, Li S, Na L, Feng R, Liu L, Li Y, Sun C. Mangiferin decreases plasma free fatty acids through promoting its catabolism in liver by activation of AMPK. PLOS One 2012; 7(1): e30782.

14. Goodarzi MO, Carmina E, Azziz R. DHEA, DHEAS and PCOS. J Steroid Biochem Mol Biol 2015; 145: 213-225.

15. Sahmay S, Aydogan Mathyk B, Sofiyeva N, Atakul N, Azemi A, Erel T. Serum AMH levels and insulin resistance in women with PCOS. Eur J Obstet Gynecol Reprod Biol 2018; 224(159-164.

16. Li MF, Zhou XM, Li XL. The Effect of Berberine on Polycystic Ovary Syndrome Patients with Insulin Resistance (PCOS-IR): A Meta-Analysis and Systematic Review. Evid Based Complement Alternat Med 2018, 2018: 2532935.

17. Yang $C Q, X u J H$, Yan $D D$, Liu BL, Liu K, Huang $F$. Mangiferin ameliorates insulin resistance by inhibiting inflammation and regulating adipokine expression in adipocytes under hypoxic condition. Chin J Nat Med 2017; 15(9): 664-673.

18. Li T, Mo H, Chen W, Li L, Xiao Y, Zhang J, Li X, Lu Y. Role of the PI3K-Akt Signaling Pathway in the Pathogenesis of Polycystic Ovary Syndrome. Reprod Sci 2017; 24(5): 646-655.

19. Wu $C$, Jiang $F$, Wei $K$, Jiang $Z$. Exercise activates the PI3K-AKT signal pathway by decreasing the expression of 5alpha-reductase type 1 in PCOS rats. Sci Rep 2018; 8(1): 7982.

20. Neisy A, Zal F, Seghatoleslam A, Alaee S. Amelioration by quercetin of insulin resistance and uterine GLUT4 and ERalpha gene expression in rats with polycystic ovary syndrome (PCOS). Reprod Fertil Dev 2018.

21. Teede H, Deeks A, Moran L. Polycystic ovary syndrome: a complex condition with psychological, reproductive and metabolic manifestations that impacts on health across the lifespan. BMC Med 2010; 8: 41.

22. Marquez L, Garcia-Bueno B, Madrigal JL, Leza JC. Mangiferin decreases inflammation and oxidative damage in rat brain after stress. Eur J Nutr 2012; 51(6): 729-739.

23. Bhaskar S, Shalini V, Helen A. Quercetin regulates oxidized $L D L$ induced inflammatory changes in human PBMCs by modulating the TLR-NF-kappaB signaling pathway. Immunobiology 2011; 216(3): 367-373.

24. Rui L, Aguirre V, Kim JK, Shulman GI, Lee A, Corbould A, Dunaif A, White MF. Insulin/IGF-1 and TNF-alpha stimulate phosphorylation of IRS-1 at inhibitory Ser307 via distinct pathways. J Clin Invest 2001; 107(2): 181 189.

25. Gonzalez F. Inflammation in Polycystic Ovary Syndrome: underpinning of insulin resistance and ovarian dysfunction. Steroids 2012; 77(4): 300-305. 